\title{
VELOCIDADE DE CRESCIMENTO, CARACTERÍSTICAS DA CARCAÇA, ANÁLISE HORMONAL E MORFOLOGIA DO EPITÉLIO INTESTINAL DE CORDEIROS SOB DOIS FOTOPERÍODOS ${ }^{1}$
}

\author{
GROWTH RATE, CARCASS TRAITS, HORMONAL LEVELS AND \\ INTESTINAL EPITHELIUM MORPHOLOGY OF LAMBS \\ FINISHED UNDER TWO PHOTOPERIODS
}

\author{
Renata de Lello Montenegro ${ }^{2}$ Edson Ramos de Siqueira ${ }^{3}$
}

RESUMO

\begin{abstract}
Com o objetivo de avaliar o efeito do fotoperíodo sobre o desenvolvimento de ovinos até o peso vivo em torno de $38-40 \mathrm{~kg}$, foram utilizados 26 cordeiros Ile de France $x$ Corriedale, divididos aleatoriamente em 2 grupos: fotoperiodo longo(18h de luz/ $6 \mathrm{~h}$ de escuridão) e fotoperíodo controle( $12 \mathrm{~h}$ de luz/ $12 \mathrm{~h}$ de escuridão). Diariamente, dados sobre consumo alimentar foram coletados e, semanalmente, os animais eram pesados e amostras de sangue eram colhidas para dosagem dos níveis plasmáticos dos hormônios Triiodotironina(T3), Tiroxina(T4) e Cortisol. Amostras de jejuno e íleo foram coletadas para análise morfométrica do epitélio intestinal, $e$ foram estudadas características da carcaça. Os cordeiros submetidos ao fotoperíodo longo, apresentaram maior consumo alimentar e maior ganho de peso $(P<0,05)$. Não se verificou diferença significativa nas concentrações plasmáticas dos hormônios estudados, na morfometria intestinal e nas características da carcaça. Concluiu-se que o fotoperíodo longo influencia, positivamente, o desempenho dos cordeiros, sendo esta prática economicamente viável.
\end{abstract}

Palavras- chave: fotoperíodo, cordeiro, crescimento, hormônios, morfologia intestinal.

\section{SUMMARY}

With the objective of testing the influence of photoperiod on development of lambs, twenty-six crossbred Ile de France $x$ Corriedale male lambs were studied slaughtered with 38 to $40 \mathrm{~kg}$ The lambs were slaughtered with 38 to $40 \mathrm{~kg}$. Animals were ramdomly divided in two groups: long photoperiod (18h light/ 6 h darkness) and control (12h light/ $12 \mathrm{~h}$ darkness). Food consumption was daily evaluated. Weekly, lambs were weighted and blood samples collected for Triiodotironine (T3), Thyroxine (T4) and Cortisol plasmatic levels determination. Jejunal and ileal samples were collected for morphometric analyses of the intestinal epithelium. Carcass characteristics were also studied. Lambs submitted to the long photoperiod have greater food consumption and live weight gain when compared to control $(P<0.05)$. No significant differences were observed on the plasmatic levels of the hormones, in the morphology of the intestinal epithelium or carcass characteristics. In conclusion, long photoperiod has a positive effect on the development of lambs, being an economically viable techinic.

Key words: photoperiod, lambs, growth, hormones, intestinal morphology.

\section{INTRODUÇÃO}

Nos Estados onde a ovinocultura é tradicional, a recria dos animais é feita em pasto. Essa prática, via de regra, é pouco eficiente no Estado de São Paulo, onde a parasitose é mais freqüente e responsável por grandes perdas no desenvolvimento dos cordeiros e também por altas taxas de mortalidade (SIQUEIRA et $\boldsymbol{a l}$. , 1993). A solução para esse problema foi o desmame precoce e confinamento total dos cordeiros até atingirem o

\footnotetext{
${ }^{1}$ Parte da dissertação de mestrado da primeira autora, apresentada ao curso de Pós-graduação em Nutrição e Exploração Animal da Faculdade de Medicina Veterinária e Zootecnia (FMVZ), Universidade Estadual Paulista (UNESP), Botucatu-SP. E-mail: montenegrolohr@ clear.net.nz Autora para correspondência.

${ }^{2}$ Médico Veterinário, Mestrando em Nutrição e Exploração Animal da FMVZ, UNESP, Botucatu- SP.

${ }^{3}$ Professor Titular do Departamento Produção e Exploração Animal da FMVZ, UNESP, Botucatu- SP .
} 
peso de abate. Hoje, buscam-se maneiras de se melhorar o desempenho dos cordeiros no confinamento.

Por ser um ruminante de reprodução sazonal, a estação reprodutiva dos animais é estimulada, principalmente, pelo decréscimo da luminosidade, acreditando-se que isso também pode influenciar o seu desempenho. Já foi observado que animais recebendo o mesmo tipo de alimento e na mesma quantidade, apresentam maiores ingestão e crescimento nos meses de verão, onde a quantidade de luz recebida é maior (BRINKLOW \& FORBES, 1984). A possibilidade de se melhorar o desempenho dos cordeiros com o acréscimo de luz durante o confinamento é muito interessante, no entanto, seus mecanismos ainda não são claros. Alterações nas concentrações plasmáticas dos hormônios Triiodotironina $\left(\mathrm{T}_{3}\right)$, Tiroxina $\left(\mathrm{T}_{4}\right)$, Prolactina, Cortisol e Hormônio do Crescimento $(\mathrm{GH})$, foram citados como possíveis responsáveis por esse incremento (FORBES et al., 1979a; BRINKLOW \& FORBES 1984; BARENTON et al., 1988 e PETITCLERC \& ZINN 1991). Como os animais expostos ao fotoperíodo longo apresentam maior ingestão de alimentos, é provável que ocorram mudanças das características morfológicas do epitélio absortivo desses animais. Por esse motivo, este estudo tem como objetivo analisar a influência de dois diferentes fotoperíodos no desempenho, nas características da carcaça, no perfil hormonal e na morfologia do epitélio intestinal de cordeiros terminados em regime de confinamento total.

\section{MATERIAL E MÉTODOS}

A coleta de dados e material dos animais foi conduzida na câmara bioclimática do Depto. de Melhoramento e Nutrição Animal da FMVZ (Faculdade de Medicina Veterinária e Zootecnia) UNESP (Universidade Estadual Paulista), Botucatu. O estudo das carcaças e da carne foi efetuado nos laboratórios de carnes do Departamento de Produção e Exploração Animal da FMVZ e Tecnologia dos Produtos Agropecuários da Fac. de Ciências Agronômicas, UNESP, Botucatu. As análises hormonais foram realizadas no laboratório de bioclimatologia da FCAV (Faculdade de Ciências Agrárias e Veterinárias) - UNESP, Jaboticabal. O material histológico foi processado no Departamento de Patologia da Faculdade de Medicina, UNESP, Botucatu e as análises morfométricas das lâminas no Departamento de Morfologia-Instituto de Biociências, UNESP, Botucatu.

Foram utilizados 26 cordeiros inteiros, mestiços Ile de France x Corriedale, com idade média de 60 dias e peso médio de $18,3 \mathrm{~kg}$, ao início do experimento. $\mathrm{O}$ delineamento foi inteiramente casualizado, com dois tratamentos e treze repetições. Os animais foram submetidos a um período de adaptação de 15 dias, (12 horas de luz e 12 horas de escuridão), durante 84 dias no verão. Após a adaptação, os cordeiros foram pesados e separados aleatoriamente em dois grupos de treze animais: FL (fotoperíodo longo) com 18 horas de luz e 6 horas de escuridão, e FC (fotoperíodo controle) com 12 horas de luz e 12 horas de escuridão. Em ambos os tratamentos as luzes eram acesas às $7 \mathrm{~h}$ e apagadas às $19 \mathrm{~h}$ no tratamento $\mathrm{FC}$ e à $1 \mathrm{~h}$ no FL. A iluminação consistiu de 8 lâmpadas fluorescentes em cada sala, com intensidade lumínica variando entre 550 e 700 lux, controlada por temporizador (Intermatic Timeall).

A ração com $16 \%$ PB e $67 \%$ de NDT, foi oferecida à vontade e dados sobre o consumo diário foram coletados. Semanalmente, os animais foram pesados e amostras de sangue foram coletadas para determinação das concentrações de hormônios $\mathrm{T}_{3}, \mathrm{~T}_{4}$ e Cortisol, coletadas. A técnica utilizada para a dosagem hormonal foi a de Análise Fluorométrica (Stratus II Immunoassay System - Baxter).

Os animais foram abatidos com peso entre $38-40 \mathrm{~kg}$ e idade média de 140 dias, e após a abertura do abdômen, coletaram-se amostras do jejuno e do íleo. No dia anterior ao sacrifício, às $14 \mathrm{~h}$ os animais eram transportados para o local de abate, onde permaneciam em jejum de aproximadamente 18 horas, com água à disposição. Após o sacrifício, o aparelho gastrintestinal era esvaziado, para obtenção do peso vivo vazio (peso vivo ao abate, menos o conteúdo do aparelho gastrintestinal), e desse modo determinar o rendimento verdadeiro (relação entre o peso da carcaça quente e o peso vivo vazio).

Uma vez terminada a evisceração, as carcaças foram pesadas (peso da carcaça quente), transportadas para a câmara fria e submetidas a resfriamento por 24 horas a $4^{\circ} \mathrm{C}$. Ao término desse período, registrou-se o peso da carcaça fria, calculando-se então o rendimento comercial (relação entre o peso da carcaça fria e o peso vivo ao abate). Para efeito de mensuração, as carcaças foram mantidas com as articulações tarso metatarsianas distanciadas em 17 centímetros, através de gancho próprio, realizando-se medidas objetivas (SAÑUDO \& SIERRA 1986) e subjetivas (COLOMERROCHER, 1988) das carcaças. Posteriormente, as carcaças foram divididas ao meio sendo a metade esquerda seccionada em 7 regiões anatômicas, segundo (COLOMER \& ESPEJO 1972); os cortes foram pesados individualmente, sendo o lombo encaminhado para o laboratório, onde permaneceu 
congelado para posterior dissecção. Através dessa, foram determinadas as proporções dos tecidos ósseo, muscular e adiposo, o qual foi subdividido em gordura subcutânea e intermuscular. Determinou-se a área transversal do músculo Longissimus dorsi e também as medidas A (largura máxima do músculo), $\mathrm{B}$ (profundidade máxima do músculo), C (espessura de gordura sobre o músculo), e J (maior espessura de gordura de cobertura no perfil do lombo).

A comparação entre os grupos foi efetuada, utilizando-se para as variáveis quantitativas, $\mathrm{o}$ teste $\mathrm{t}$ para duas amostras independentes, com a determinação da média para indicação da tendência central; para as variáveis semi-quantitativas (notas), a Prova Não Paramétrica de Mann-Whitney, com a determinação da mediana para indicação da medida de tendência central (ZAR, 1984).

\section{RESULTADOS E DISCUSSÃO}

Foram detectadas diferenças significativas no ganho de peso $(\mathrm{p}<0,01)$, no consumo $(\mathrm{p}<0,05)$ e na conversão alimentar $(\mathrm{p}<0,05)$ dos cordeiros (Tabela 1), com superioridade dos animais submetidos ao FL. Esses resultados corroboram os estudos feitos por FORBES et al. (1979a); SCHANBACHER \& CROUSE (1980); SCHANBACHER et al. (1982); BRINKLOW \& FORBES (1984); SCHANBACHER (1984); e BARENTON et al. (1988).

O mecanismo pelo qual a luminosidade afeta o desempenho dos animais não é claro. Ainda se questiona se o animal tem um aumento no consumo alimentar devido ao maior crescimento, ou se tem um melhor ganho de peso devido apenas a um aumento de apetite. O comprimento da perna e o perímetro da garupa dos animais submetidos à longa luminosidade foram superiores $(\mathrm{p}<0,05)$ aos dos animais submetidos ao FC (Tabela 3), podendo, dessa maneira, explicar o melhor desempenho dos cordeiros sob FL. BRINKLOW \& FORBES (1984) e PETITCLERC \& ZINN (1991) também constataram efeito da quantidade de luz sobre as carcaças de cordeiros expostos a dias longos, pois estas apresentaram maior comprimento e maior largura do que aquelas dos cordeiros submetidos a dias curtos.

Diferença significativa dos níveis plasmáticos de $\mathrm{T}_{3}$ e $\mathrm{T}_{4}$ entre os grupos foi observada (Tabela 2), somente no primeiro momento de colheita, para o hormônio $\mathrm{T}_{3}$; e isso pode ter sido causado por algum efeito não controlado antes da colheita. Devido a flutuações cíclicas na secreção desses hormônios, é possível que o horário escolhido para a colheita do material, tenha sido o responsável pela inobservância de diferenças entre os grupos, para essa variável. FORBES et al. (1979b) e LINCOLN et $\boldsymbol{a l}$. (1982) não observaram variações sazonais nas concentrações plasmáticas de $\mathrm{T}_{3}$ e $\mathrm{T}_{4}$; por outro lado, LINCOLN et al. (1980) afirmaram que os níveis plasmáticos de $\mathrm{T}_{3}$ e $\mathrm{T}_{4}$ foram maiores, quando os animais estiveram expostos ao FL.

FITZGERALD et $\boldsymbol{a l}$. (1982) verificaram que os hormônios tiroidianos estavam aumentados nos ovinos expostos à longa luminosidade, devido à maior ingestão de alimentos e não ao fotoperíodo. Durante a primavera e verão, quando se tem uma oferta maior de alimentos, os níveis plasmáticos de $\mathrm{T}_{3}$ e $\mathrm{T}_{4}$ são maiores do que no inverno. Porém, isso não se observou quando esses animais receberam alimentação restrita. Dessa maneira, pode-se suspeitar, que o FL desencadeie algum mecanismo neuroendócrino que aumente o apetite. $\mathrm{O}$ animal, tendo uma quantidade maior de energia, ativa a hipófise que secreta o hormônio estimulante da tireóide (TSH), que vai aumentar a secreção dos hormônios tiroidianos, responsáveis pelo aumento da atividade metabólica de todos os órgãos, resultando em um maior desempenho. Especula-se que o FL diminua a secreção do cortisol; com isso, ocorre um declínio do catabolismo das proteínas, explicando-se dessa maneira, o melhor desempenho dos animais sob longa luminosidade.

Não se observou diferença significativa da concentração plasmática do cortisol, em 4 momentos entre os dois grupos (Tabela 2). Esses resultados discordam dos de BRINKLOW \&

Tabela 1 - Medias e desvios padrão do ganho de peso diário ( $\mathrm{kg} / \mathrm{dia})$, consumo alimentar médio (kg/dia), conversão alimentar e tempo para atingir o peso de abate (dias) de cordeiros submetidos a fotoperíodo longo (FL) e controle (FC). Prova não paramétrica de MannWhitney para comparação de tratamento.

\begin{tabular}{ccccc}
\hline Tratamento & Ganho médio diário kg/dia & Consumo alimentar kg/dia & Conversão alimentar & Tempo para atingir peso de abate (dias) \\
\hline FL & $0,275 \pm 0,003$ & $1,341 \pm 0,170$ & $4,752 \pm 0,026$ & $77,400 \pm 8,560$ \\
FC & $0,233 \pm 0,003$ & $1,281 \pm 0,160$ & $5,397 \pm 0,032$ & $84,100 \pm 5,470$ \\
Diferença & 0,042 & 0,060 & 0,645 & 6,7 \\
Estatítica & $\mathrm{T}=2,891(\mathrm{p}<0,01)$ & $\mathrm{T}=2,028(\mathrm{p}<0,05)$ & $\mathrm{T}=2,141(\mathrm{p}<0,05)$ & $\mathrm{T}=2,087(0,05<\mathrm{p}<0,10)$ \\
\hline
\end{tabular}


Tabela 2 - Medianas dos níveis plasmáticos dos hormônios: Cortisol ( $\mu \mathrm{g} / \mathrm{d} \ell)$, T3 (ng/m $\ell$ ) e T4 ( $\mu \mathrm{g} / \mathrm{d} \ell)$ em quatro momentos de amostragem de cordeiros submetidos a fotoperíodo longo (FL) e controle (FC). Prova não paramétrica de Mann-Whitney para comparação de tratamento.

\begin{tabular}{lcccccccc}
\hline \multirow{2}{*}{ Parâmetros } & \multicolumn{2}{c}{$22 / 11 / 96$} & \multicolumn{2}{c}{$12 / 12 / 96$} & \multicolumn{2}{c}{$26 / 12 / 96$} & \multicolumn{2}{c}{$16 / 01 / 97$} \\
\cline { 2 - 9 } & $\mathrm{FL}$ & $\mathrm{FC}$ & $\mathrm{FL}$ & $\mathrm{FC}$ & $\mathrm{FL}$ & $\mathrm{FC}$ & $\mathrm{FL}$ & $\mathrm{FC}$ \\
\hline Cortisol $(\mu \mathrm{g} / \mathrm{d} \ell)$ & 5,9 & 6,0 & 5,2 & 5,2 & 4,0 & 4,0 & 5,7 & 5,1 \\
Estatística & $\mathrm{U}=69$ & $(\mathrm{P}>0,10)$ & $\mathrm{U}=81$ & $(\mathrm{P}>0,10)$ & $\mathrm{U}=72$ & $(\mathrm{P}>0,10)$ & $\mathrm{U}=78,5$ & $(\mathrm{P}>0,10)$ \\
T3 $(\mathrm{ng} / \mathrm{m} \ell)$ & 0,83 & 0,58 & 0,64 & 0,83 & 0,96 & 0,83 & 0,87 & 0,86 \\
Estatística & $\mathrm{U}=42$ & $(\mathrm{P}>0,10)$ & $\mathrm{U}=57$ & $(\mathrm{P}>0,10)$ & $\mathrm{U}=51$ & $(\mathrm{P}>0,10)$ & $\mathrm{U}=73,5$ & $(\mathrm{P}>0,10)$ \\
T4 $(\mu \mathrm{g} / \mathrm{m} \ell)$ & 6,1 & 5,2 & 5,6 & 6,1 & 5,8 & 5,8 & 5,7 & 5,2 \\
Estatística & $\mathrm{U}=67$ & $(\mathrm{P}>0,10)$ & $\mathrm{U}=51,5$ & $(\mathrm{P}>0,10)$ & $\mathrm{U}=66$ & $(\mathrm{P}>0,10)$ & $\mathrm{U}=84$ & $(\mathrm{P}>0,10)$ \\
\hline
\end{tabular}

FORBES (1984), que afirmaram que cordeiros expostos ao FL, apresentaram níveis plasmáticos de cortisol mais baixos que cordeiros sob FC. Esses dados conflitantes podem ser explicados pelo estresse dos animais durante a colheita de sangue, que ocasionam picos na secreção do cortisol, o qual pode ter mascarado possíveis diferenças basais.

A ingestão de alimentos também sofre influência do fotoperíodo. Animais sob longa luminosidade apresentam maior ingestão (FORBES et al., 1979a; RYG \& JACOBSEN 1982; SCHANBACHER $\boldsymbol{e}$ t al., 1982; SØRENSEN 1984), a qual pode induzir a uma hipertrofia do epitélio intestinal, um dos mecanismos através dos quais o intestino se adapta à maior oferta de alimentos (FERRARIS 1994). No presente estudo o peso vazio do aparelho gástrico dos cordeiros sob FL foi maior $(\mathrm{p}<0,05)$ do que nos cordeiros sob FC (Tabela 3), corroborando FORBES et al. (1979a). O maior peso desse órgão pode justificar a maior capacidade de ingestão dos animais. Estudos mais detalhados, como, por exemplo, análise morfométrica dos pré estômagos e estômago, devem ser feitos a fim de se obterem informações sobre o possível papel desses órgãos no melhor desempenho dos cordeiros sob FL. $\mathrm{O}$ peso do intestino vazio teve a tendência de ser maior $(0,05<\mathrm{p}<0,10)$ nos cordeiros sob FL (Tabela 3), corroborando EISEMANN et al. (1984), os quais observaram diferença significativa. A altura e a área das vilosidades intestinais não diferiram significativamente entre os grupos (Tabela 3) e apenas o íleo foi avaliado, uma vez que o jejuno apresentou-se autolizado. É importante que continuem novas pesquisas nessa área, a fim de esclarecer a possível relação do epitélio intestinal e o melhor desempenho de animais sob o FL.

Conforme demonstra a tabela 3 , o fotoperíodo não influenciou as características das carcaças (exceto comprimento da perna e perímetro da garupa), corroborando FORBES et al. (1979a) e contrariando SCHANBACHER \& CROUSE (1980). Todavia, estes sacrificaram os cordeiros com diferentes pesos, que pode ter representado fator de confundimento, já que esta variável, mais do que a idade, afeta sobremaneira a qualidade da carcaça (OSÓRIO, 1992). Analisando os rendimentos das carcaças, os resultados foram superiores aos obtidos por FERNANDES (1994), que optou por peso ao abate de $32 \mathrm{~kg}$. Tal fato foi decorrente dos maiores teores de gordura encontrados na presente pesquisa, demonstrados pela porcentagem de gordura do lombo, medidas C e J. O efeito do nível de tecido adiposo sobre o aumento da rendimento foi também constatado por KIRTON \& BARTON (1962), ESPEJO \& COLOMER- ROCHER (1971), CAÑEQUE et al. (1989) e DELFA et al. (1991).

Os níveis de gordura poderiam ter sido inferiores, caso o abate tivesse ocorrido a pesos menores. SIQUEIRA et al. (1998) obtiveram $11,60 \%$ de gordura, $61,50 \%$ de músculo e $21,70 \%$ de osso, com pesos ao sacrifício na faixa de 28 a $32 \mathrm{~kg}$. Sob o ponto de vista econômico, verificou-se a tendência dos cordeiros mantidos sob FL, terem auferido melhor resultado, pelo fato de terem atingido o peso de abate préestabelecido, 7 dias antes do grupo controle. Este fato redundou em economia de $8,97 \mathrm{~kg}$ de ração por cordeiro no período. Por outro lado, o custo adicional de energia elétrica foi equivalente a $4,0 \mathrm{~kg}$ de ração. Em suma, houve uma economia de $4,97 \mathrm{~kg}$ de ração por cordeiro doFL.

\section{CONCLUSÕES}

A utilização de iluminação artificial nos confinamentos, visando ao aumento do fotoperíodo para 18 horas, é uma prática recomendada, pois propicia um incremento no ganho de peso e no consumo, diminuindo o tempo de confinamento necessário para os cordeiros atingirem o peso de 
Tabela 3 - Médias e desvios padrão das características da carcaça, morfometria do epitélio intestinal e das medidas tomadas do corte comercial do lombo, peso (g) e proporções (\%) dos tecidos adiposo, muscular e ósseo de cordeiros sumetidos a fotoperíodo longo (FL) e fotoperíodo controle (FC).

\begin{tabular}{lccc}
\hline & \multicolumn{1}{c}{ Parâmetros } & $\mathrm{FL}$ & Estatística \\
\hline Rendimento comercial $(\%)$ & $45,26 \pm 1,95$ & $44,57 \pm 1,33$ & $\mathrm{~T}=0,928(\mathrm{P}>0,25)$ \\
Rendimento verdadeiro $(\%)$ & $53,40 \pm 1,47$ & $52,73 \pm 1,22$ & $\mathrm{~T}=1,112(\mathrm{P}>0,25)$ \\
Peso da carcaça quente $(\mathrm{kg})$ & $16,79 \pm 0,77$ & $16,23 \pm 0,62$ & $\mathrm{~T}=1,794(0,05<\mathrm{P}<0,10)$ \\
Peso da carcaça fria $(\mathrm{kg})$ & $16,34 \pm 0,81$ & $15,75 \pm 0,66$ & $\mathrm{~T}=1,804(0,05<\mathrm{P}<0,10)$ \\
Comprimento da perna $(\mathrm{cm})$ & $31,11 \pm 0,49$ & $30,60 \pm 1,64$ & $\mathrm{~T}=2,359(\mathrm{P}<0,05)$ \\
Perímetro da garupa $(\mathrm{cm})$ & $58,43 \pm 1,58$ & $55,95 \pm 2,71$ & $\mathrm{~T}=2,500(\mathrm{P}<0,05)$ \\
Peso do intestino $(\mathrm{kg})$ & $1,48 \pm 0,18$ & $1,36 \pm 0,08$ & $\mathrm{~T}=2,003(0,05<\mathrm{P}<0,10)$ \\
Peso do aparelho gástrico(kg) & $1,54 \pm 0,11$ & $1,39 \pm 0,11$ & $\mathrm{~T}=3,080(\mathrm{P}<0,01)$ \\
Morfometria altura $(\mu)$ das microvilosidades intestinais & $894,61 \pm 230,16$ & $870,15 \pm 225,06$ & $\mathrm{~T}=0,170(\mathrm{P}>0,5)$ \\
Morfometria área $\left(\mu^{2}\right)$ microvilosidades intestinais & $71381,07 \pm 12214,88$ & $85450,99 \pm 22317,51$ & $\mathrm{~T}=1,302(\mathrm{P}>0,25)$ \\
Medida A $(\mathrm{cm})$ & $5,51 \pm 0,28$ & $5,45 \pm 0,25$ & $\mathrm{~T}=0,504(\mathrm{P}>0,50)$ \\
Medida B $(\mathrm{cm})$ & $3,20 \pm 0,20$ & $3,04 \pm 0,23$ & $\mathrm{~T}=1,652(\mathrm{P}>0,25)$ \\
Medida C $(\mathrm{mm})$ & $3,46 \pm 1,22$ & $2,75 \pm 0,85$ & $\mathrm{~T}=1,511(\mathrm{P}>0,25)$ \\
Medida $(\mathrm{mm})$ & $9,98 \pm 2,19$ & $8,68 \pm 2,77$ & $\mathrm{~T}=1,163(\mathrm{P}>0,25)$ \\
AOL $\left(\mathrm{cm}^{2}\right)$ & $11,89 \pm 1,26$ & $12,96 \pm 1,64$ & $\mathrm{~T}=1,428(\mathrm{P}>0,25)$ \\
Gordura peso $(\mathrm{g})$ & $227,81 \pm 65,48$ & $206,89 \pm 43,71$ & $\mathrm{~T}=0,845(\mathrm{P}>0,25)$ \\
\multicolumn{1}{c}{$\%$} & $27,67 \pm 6,40$ & $25,88 \pm 5,20$ & $\mathrm{~T}=0,685(\mathrm{P}>0,50)$ \\
Músculo peso $(\mathrm{g})$ & $425,65 \pm 44,21$ & $425,97 \pm 61,44$ & $\mathrm{~T}=0,002(\mathrm{P}>0,50)$ \\
\multicolumn{1}{c}{$\%$} & $52,79 \pm 5,71$ & $53,29 \pm 6,18$ & $\mathrm{~T}=0,185(\mathrm{P}<0,50)$ \\
Osso peso $(\mathrm{g})$ & $110,86 \pm 20,12$ & $123,49 \pm 40,88$ & $\mathrm{~T}=0,877(\mathrm{P}>0,50)$ \\
\multicolumn{1}{c}{$\%$} & $13,12 \pm 4,03$ & $15,07 \pm 3,79$ & $\mathrm{~T}=1,129(\mathrm{P}<0,50)$ \\
\hline
\end{tabular}

abate, sem acarretar alterações na qualidade das carcaças. Novos estudos sobre os mecanismos pelos quais o fotoperíodo influencia o consumo e o ganho de peso dos cordeiros devem ser realizados.

\section{REFERÊNCIAS BIBLIOGRAFICAS}

BARENTON, B., RAVAULT, J.P., CHABANET, C., et al. Photoperiodic control of growth hormone secretion and body weight in rams. Domestic Animal Endocrinology, v.5, n.3, p.247-255, 1988 .

BRINKLOW, B.R., FORBES, J.M. Manipulation of growth in farm animals. The Netherlands: Roche \& O'Callaghan, 1984. p.260-273.

CAÑEQUE, N.D., HUILDOBRO, F.R., DOLZ, J.F., et al. Produção de carne de cordeiro. Madrid : Ministério de Agricultura Pesca y Alimentacion, 1989. 520p.

COLOMER-ROCHER, F. Estudio de los parametros que definen los caracteres cuantitativos y cualitativos de las canales. In: CURSO INTERNACIONAL SOBRE PRODUCCIÓN DE CARNE Y LECHE CON BASES EN PASTOS Y FORRAJES. 1988, España. S.1 : s.e, 1998. 108p.

COLOMER-ROCHER,F., ESPEJO, M.D. Determinación del peso óptimo de sacrifício de los corderos procedentes del cruzamiento Manchego X Rasa Aragonesa en función del sexo. Revista Itea, p. 219-225, 1972.

DELFA, R., GONCALÉZ, C. El quinto cuarto. Revista Ovis, n. 17, p.48-68, 1991
EISEMANN, J.H., BAUMAN, D.E., HOGUE, D.E., et al. Evaluation of a role for prolactin in growth and the photoperiod-induced growth response in sheep. Journal of Animal Science, v.59, n.1, p.86-94, 1984.

ESPEJO, M.D., COLOMER-ROCHER, F. Influencia del estado de engrasamiento y conformacion sobre el porcentaje de piezas de la canal ovina. INIA/ Ser Prod Anim, n.1, p77-92, 1971.

FERNANDES, S. Peso vivo ao abate e características da carcaça de cordeiros da raça Corriedale e mestiços Ile de France $x$ Corriedale, recriados em confinamento. Botucatu-SP.1994. p.82. Dissertação (Mestrado em Zootecnia) - Curso de Pós-graduação em Nutrição e Produção Animal, Universidade Estadual Paulista, 1994.

FERRARIS, R.P. Physiology of the gastrointestinal tract. 3.ed. New York : Leonard R. \& Johnson Raven, 1994. Cap 54: Regulation of intestinal nutrient transport: p. 1821-1840.

FITZGERALD, J., MICHEL, F., BUTLER, W.R. Growth and sexual maturation in ewes: The role of photoperiod, diet and temperature on growth rate and the control of prolactin, thyroxine and luteinizing hormone secretion. Journal of Animal Science, v.55 n.6, p.1431-1440, 1982.

FORBES, J.M., EL SHAHAT, A.A., JONES, R., et al. The effect of daylengthy on the growth of lambs 2 . Blood concentration of growth hormone, prolactin, insulin and thyroxine, and the effect of feeding. Animal Production, v.29, p.43-51, 1979a. 
FORBES, J.M., EL SHAHAT, A.A., JONES, R., et al. The effect of daylenght on the growth of lambs 1. Comparisons of sex, level of feeding, shearing and breed of sire. Animal Production, v.29, p.33-42, 1979b.

KIRTON, A.H., BARTON, R.A. Study of some indices of the chemical composition of lamb carcasses. Journal of Animal Science, v.21, p.535-557, 1962.

LINCOLN, G.A., KLANDORF, H., ANDERSON, N. Photoperiodic control of thyroid function and wool and horn growth in rams and the effect of cranial sympathectomy. Endocrinology, v.107,n.5, p.1543-1548, 1980.

LINCOLN,G. A., ALMEIDA, O.F., et al. Hourly fluctuations in the blood leves of melatonin, prolactin, luteinizing hormone, follicle-stimulating hormone, testosterone, tri-iodothyronine, thyroxine and cortisol in rams under artificial photoperiods, and the effects of cranial sympathectomy. Journal of Endocrinology, v.92, p.237-250, 1982.

OSÓRIO, J.C.S. Estudio de la calidad de canales comercializadas en el tipo Ternasco según la procedencia: bases para la mejora de dicha calidad en Brasil. Zaragoza - España, 1992. 335p. Tese (Doutorado em Veterinária) Faculdad de Veterinaria, Universidad de Zaragoza, 1992.

PETITCLERC, D., ZINN, S. Effects of photoperiod on growth and development of ruminants. Advances in Meat Research, v.7, p. 427-453, 1991.

RYG , M., JACOBSEN, E. Seasonal changes in growth rate, feed intake, growth hormone, and thyroid hormones in young male reindeer. Canadian Journal Zoology, v.60, p.2941-2946, 1982.

SAÑUDO, C., SIERRA, I. Calidad de la canal en la especie ovina. Ovino, n.1, p.127-153, 1986.
SCHANBACHER B.D., CROUSE J.D. Growth and performance of growing-finishing lambs exposed to long or short photoperiods. Journal of Animal Science, v. 51 n.4, p.943948, 1980 .

SCHANBACHER B.D., HAHN G.L., NIENABER J.A. Photoperiodic influences on performance of market lambs. Proceedings of the second international livestock environment symposium, Ames, v. 20-23, p.400-405, 1982.

SCHANBACHER B.D. Hormonal and photoperiodic control of growth. In: ROCHE, J.F., O'CALLAGHAN, D. (Eds). Manipulation of growth in farm animals. The Netherlands, 1984. p.275- 286.

SØRENSEN, M.T. Photoperiodic effect on growth and feed consumption of young bulls. In: ROCHE, J.F., O'CALLAGHAN, D. (Eds). Manipulation of growth in farm animals. The Netherlands, 1984. p.289-299.

SIQUEIRA, E.R., AMARANTE, A.F. T., FERNANDES, S. Estudo comparativo da recria de cordeiros em confinamento e pastagens. Revista Veterinária Zootecnia, v.5, p.17-28, 1993.

SIQUEIRA, E.R., FERNANDES, S., MESQUITA, V.S., $\boldsymbol{e} \boldsymbol{t}$ al. Efeito do peso ao abate sobre a eficiência de produção de cordeiros da raça Hampshire Down terminados em confinamento. In: REUNIÃO ANUAL DA SOCIEDADE Brasileira de ZoOtecnia, 35, 1998, Botucatu. Anais... Botucatu : SBZ, 1998. p.705-705.

ZAR, J.H. Biostatistical analysis. 2. ed. Euglewood Cliffs, Prentice-Hall, 1984. 718p. 